\title{
Extensive Analyses of Superconducting Cables 3D Geometry with Advanced Tomographic Examinations
}

\author{
L. Zani, M. Chiletti, D. Dumitru, M. Lungu, I. Tiseanu, F. Topin
}

\begin{abstract}
In the framework of activities embedding magnet design and associated $R \& D$ activities and relying on Cable in Conduit Conductors (CICC) technology, the singularity of the concept can rise some challenges versus their modelling in operation. Indeed, CICC includes thousands of superconducting strands, twisted together under a multi-staged scheme that also includes deformation by cabling and compaction during manufacture. This causes the accurate predictability of strands position in CICC extremely difficult, while it can be a key element for modelling their performances in operation. As a matter of fact the coupling losses rely on the CICC capability to establish interstrand shielding currents, driven by the inter-strand contacts mapping which are only accessible in prediction via accurate 3D strand trajectories geometry. Same applies for prediction of CICC mechanical properties (deformation of $\mathrm{Nb}_{3} \mathrm{Sn}$ strands) and hydraulic properties (helium coolant force-flowed between the strands), making those investigations of high added-value.
\end{abstract}

In this context, INFLPR installed a new set-up dedicated to micro-tomography that is able to examine CICCs with high resolution, allowing to get an overall overall 3D overview regarding picture of the strands location. CEA and INFLPR further developed a post-processing method to reconstruct the strands trajectories with high accuracy. The measurement and data analysis workflow was applied to two middle-size CICCs with variable void fraction from which statistics of contacts were issued. The obtained database was exploited to reconstruct equivalent 3D resistive network, in view of interpreting coupling losses tests with help of analytic CEA model (COLISEUM) based on multi-stage representation.

The above applications using CICC topology database will be discussed and tentatively compared to experimental AC losses database conducted at CEA. The outcomes will be discussed and the subsequent guidelines for future work presented.

Index Terms - nuclear fusion, CICC, superconducting magnets, tomography.

\section{INTRODUCTION}

$\mathrm{T}$ HE magnets operating in fusion tokamaks are of particular importance, due to their major role regarding the robustness of tokamak performances, especially considering their integration in the future power production mix. As a consequence of their status of merits drivers, the magnets are continuously investigated either on R\&D or on more upstream grounds. In fusion domain the Cable-in-Conduit Conductors (CICC) technology [1] is routinely considered in the magnet systems of actual tokamaks ([2][3][4]). While providing assessed advantages (among other, coolant confinement, efficient strands wetting, solid structural reinforcement...) the concept remains challenging regarding its accurate modelling using its design characteristics only towards predicting

Manuscript received 27 October 2020.

L. Zani (corresponding author, CEA-IRFM, F-13108 Saint-Paul-LezDurance, France; phone: +33442254967; fax: +33442252661; e-mail: louis.zani@cea.fr). M Chiletti is with CEA-IRFM, France. performances in tokamak. The difficulty mainly comes from CICC structure, i.e. several hundreds of millimetre-scale superconducting strands twisted together in sequence of stages and finally radially compacted to nominal dimensions. The strands positioning in CICC is difficult to predict while it is a strong driver in many CICC performances domains during operation. For example it plays an important role in electromechanical (strands movement under magnetic load), $\mathrm{AC}$ losses (inter-strands inductive network under transient magnetics), thermal stability (solid-fluid interaction in noncontinuous medium) for the most important fields.

In this framework we investigated a methodology that allows to accurately reconstruct the cable geometry in its "as-built" configuration (impossible using destructive methods) by applying dedicated tomographic examinations, imaging analyses and ad-hoc data post-processing.

This method is applied on samples of same type as JT-60SA TF conductor [5] but compacted at different rates. It is expected to extract from this study valuable qualitative and quantitative information to further generate an input for modelling tools related to the above-mentioned CICC performances domains.

\section{Generalities on Tools And Methods}

\section{A. Experimental}

\section{1) Tomographic setup}

An INFLPR tomographic facility with $225 \mathrm{kV}$ irradiation head was formerly used for this type of CICC [6] and allowed conducting studies on CICC relevant characteristics and performances (see [7] and [8]). In the present analysis the used set-up is a newly commissioned high penetration power microtomograph based on a $320 \mathrm{kV}$ reflection tube capable for increasing images resolution and contrast capacities (see in Fig. 1 and more details in [9]).

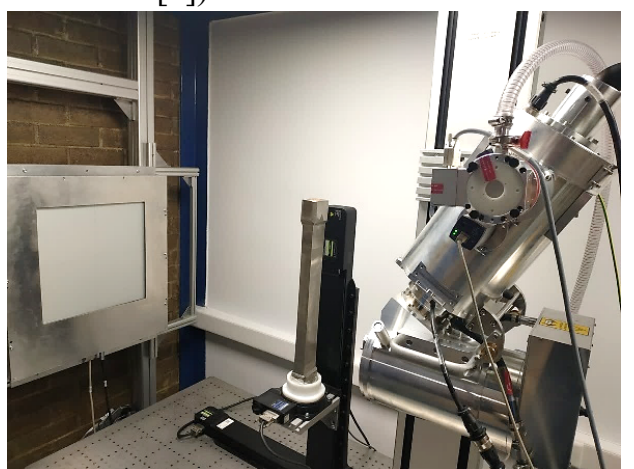

Fig. 1. High penetration power installation, with sample installed (center)

I. Tiseanu, D. Dumitru and M. Lungu are with INFLPR, 077125 Magurele, Romania.

F. Topin is withAix Marseille Université, CNRS, IUSTI UMR 7343, 13453, Marseille, France 


\section{2) CICC samples}

The CICC samples have the same cable pattern as JT-60SA TF conductor and a variable void fraction. They were issued from a study regarding impact of void fraction on hydraulic properties [10]. The used two samples are labelled "MAG42-2" and "MAG42-3" with corresponding void fractions of $33.2 \%$ and $31.6 \%$. Samples length is $\sim 300 \mathrm{~mm}$ allowing a complete last stage twist pitch to be analyzed, and therefore sample to be relevant on the point of view of strands transposition visibility.

\section{B. Data analyses methods}

\section{1) Images post-treatment}

Images of high quality are obtained, with particularly improved contrast (see comparative illustrations in Fig. 2).
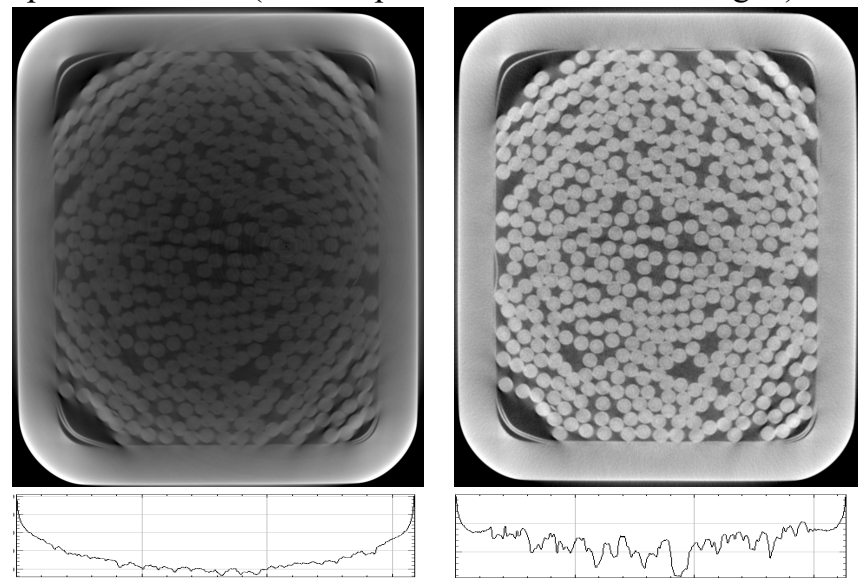

Fig. 2. MAG42-2 cross sections with $225 \mathrm{kV}$ (left) and $320 \mathrm{kV}$ (right) tomographic setup. Line profiles beneath sections are contrast-related and show the efficiency of beam-artefact mitigation in higher penetration power scan.

The resulted high-quality images permitted the application of image processing algorithms and to automatically detect using Hough transform the centroid position for each of the 486 strands ( $0.81 \mathrm{~mm}$ diameter). In order to improve the accuracy, images are aligned to recover possible sample deformations (bending, torsion). This resulted for MAG42-2 examination a post-processing of $53282 \mathrm{D}$ slices, with a distance between slices of $\sim 56 \mu \mathrm{m}$, and cross-section pixel size of $13.4 \mu \mathrm{m}$. This allowed to follow strands identity between slices and recover their trajectories. Finally, the 2D positions for all strands centres are saved as a pixel unit matrix.

\section{2) Geometrical data post-treatment}

A proximity analysis is conducted in each slice : a $2 \mathrm{D}-$ Voronoï mesh is constructed using centers as seeds and the dual Delaunay mesh (relating only for neighbor centers) is obtained [11]. Inter-centers distances map is calculated and contacting strands are identified by simple thresholding (here set at 31 pixels), as illustrated in Fig. 3. The index accounting for contacts quality is contact width, here expressing the length of the chord formed by the two intersection points of the two strands circumscribed circles.

Data were treated for in plane analysis such as contact or neighbor number distributions associated with centers or slice, but we also carried out 3D strand analyses, checking trajectories consistency (cos teta check, segregation at extremities). Also, data were used to extract multiplets (see below). At the end, properties comparisons between samples, (e.g. measured trends versus calculated trends) are also provided.
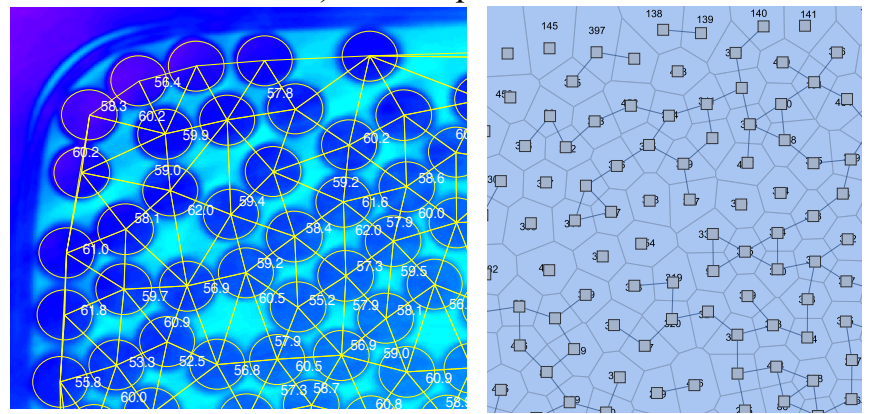

Fig. 3. Left: zoom Delaunay network superimposed on a cable section image. Only distances between strands in contact are shown (unit is pixels). Right : Delaunay limited to contacting strand and Voronoï (arbitrary location).

\section{CICC TESTS AND ANALYSES RESULTS}

\section{A. Tomographic examinations}

The two samples MAG42-2 and MAG42-3 were tested, resulting in a respective total of 5328 and 5144 slices images. During the processing workflow a data fraction (1/3) was treated along indications in section II.B.1), as preserving strands identity while allowing optimal effort efficiency. Besides, for the sake of files size optimization, downsampling on longitudinal direction (axis of the conductor) was done by averaging the gray values of 5 consecutive pixels, allowing to use a 8-bit format and rending the pixel size equal to $28 \mu \mathrm{m}$.

\section{B. Analysis of CICCs $3 D$ geometry \\ 1) $\operatorname{Cos} \theta$ check}

The $\cos \theta$ factor is the ratio between the average strands length and the sample straight length. MAG42-3 sample was analysed, chosen for its relevancy to JT-60SA TF conductor production for which information is available. As a result, the strands length statistic distribution is shown in Fig. 4.

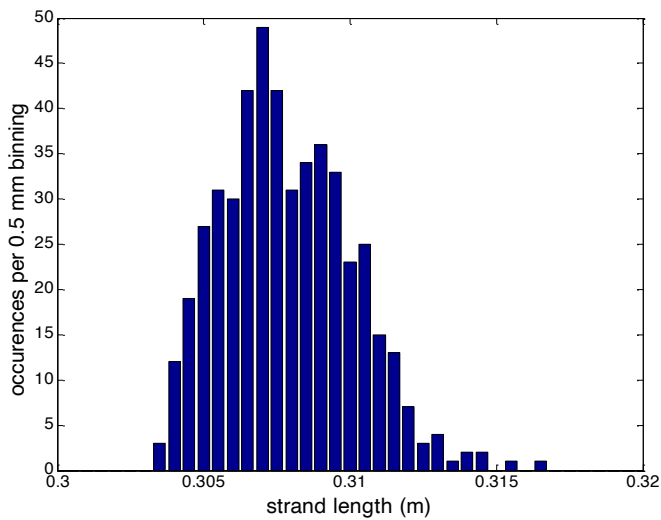

Fig. 4. MAG42-2 strands lengths statistics (binning is $0.5 \mathrm{~mm}$ )

Strands length average is $310.2 \mathrm{~mm} \pm 3 \mathrm{~mm}$, corresponding to a $\cos \theta$ of $0.974 \pm 0.007$, which is in line with the JT-60SA $\mathrm{TF}$ conductor manufacturing value, reported during production to be between 0.97 and 0.98 . This consolidates the reliability of our data post-treatment method as described in section B.

\section{2) Segregation check}

Another important consistency check was also conducted at 
strands scale. The $\mathrm{NbTi}$ and pure copper strands can be distinguished by direct visualization at both sample ends. This allows spotting each strands nature at one end and check if their calculated trajectory shows similar strand nature at the other end. We arbitrarily selected on a picture of one end 66 strands (44 $\mathrm{NbTi}+22$ copper) so $\sim 15 \%$ from the total number. Each strand identity is flagged at the first slice and its position is spotted at the opposite end (last slice) and reported on the picture of the sample opposite end, checking the nature ( $\mathrm{NbTi}$ or copper). The verification process is illustrated in Fig. 5.
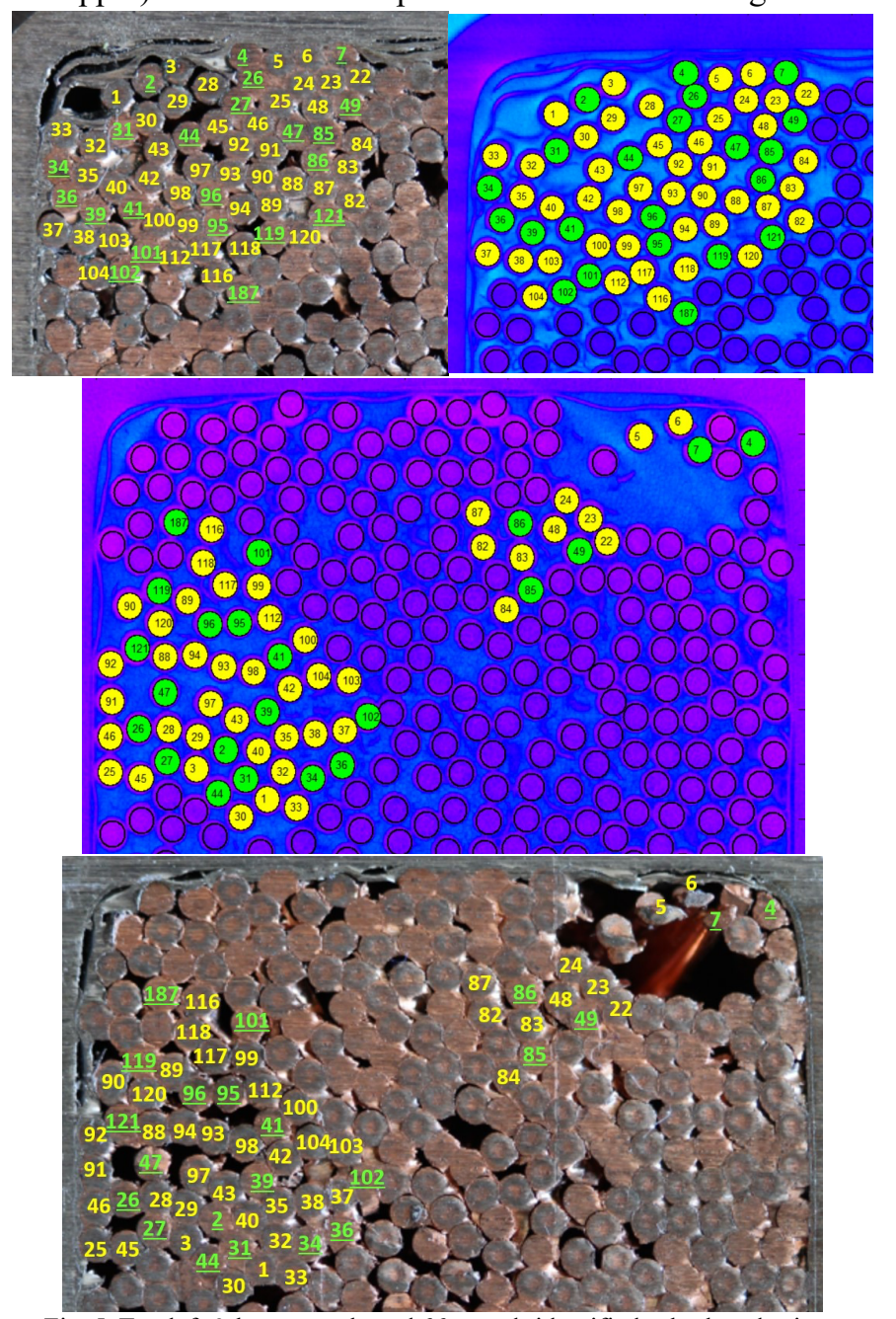

Fig. 5. Top left \& bottom: selected 66 strands identified at both ends pictures. Top right \& middle: tomography-issued strands positions at respectively first and last slice. $\mathrm{NbTi}$ and copper color code is repectively yellow and green (underlined numbers for copper labels).

It can be observed a $100 \%$ agreement, thus further consolidating the overall workflow process reliability.

\section{3) Contacts statistics}

As mentioned in Section II.B.2) the contact quality index (contact width) was compiled over the 1058 and 1033 slices respectively treated for MAG42-2 and MAG42-3 CICC samples. The inter-strands contact width distribution over the whole database was defined. The number of contacts was rescaled versus the number of samples slices to ensure consistency. Overall results can be seen in Fig. 6, showing two distributions in comparable range but somehow shifted. MAG2-3 is located at higher widths, consistently with expectations as being more compacted (lower void fraction) the implying that the strands are more pushed against each other and resulting into bigger contact areas (i.e. contact widths).

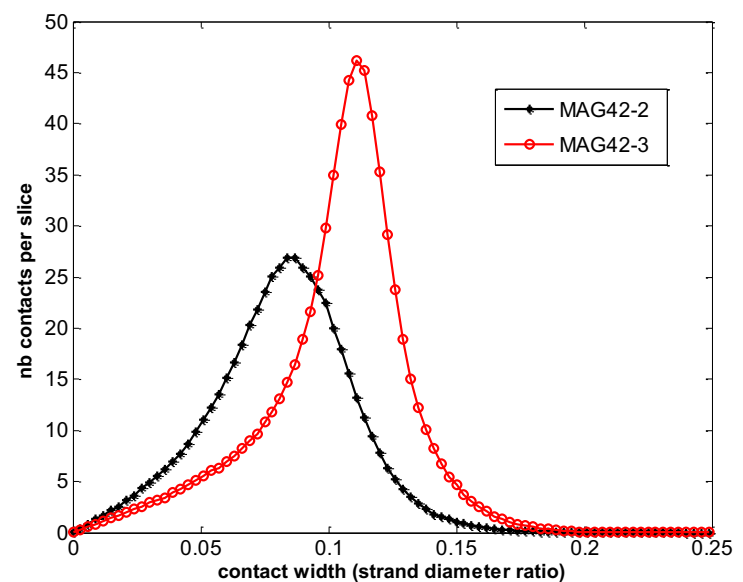

Fig. 6. MAG42-2 and MAG42-3 contact width statistics $\left(3 \cdot 10^{-3}\right.$ binning)

On quantitative ground, evaluation can be seen in Table I. Table I

CONTACT WIDTH (\% OF STRAND DIAMETER)

\begin{tabular}{lcc}
\hline \hline Sample & Average & Standard deviation. \\
\hline MAG42-2 & $8.15 \%$ & $\pm 2.7 \%$ \\
MAG42-3 & $10.29 \%$ & $\pm 2.8 \%$ \\
\hline
\end{tabular}

Contact width values (in pixel) for the tested CICC samples.

Ones can observe that the width average is about $26 \%$ higher in MAG42-3 sample, while standard deviations are comparable. Assuming a simple relation between contact width and conductance, this gives a reference for comparative analysis with AC losses tests (see section IV).

\section{4) Identification of multiplets}

The identification of assembled multiplets at all stages is important as it can bring interesting elements for CICC multiscale simulation inputs (e.g. CEA coupling losses model COLISEUM [12][13]), therefore an effort was dedicated to identifying CICC first stage triplets. Contacts statistics were further extended by identifying for each strand the ones which are the most in contact with it along its trajectory. By means of a statistical approach, it was possible to address the composition (by strand numbers) of 162 most probable triplets of the CICC. The validity of the obtained output is presented in next section.

\section{Comparative Analyses}

\section{A. Correlation with coupling losses properties}

The contact widths of section III.B.3), naturally associated in 3D with inter-strand contact surfaces, are linked with CICC inter-strand conductance, major driver of CICC coupling losses. For modelling purpose MAG42-2 and MAG42-3 samples coupling losses test were conducted at CEA in JOSEFA facility (see [14]). The resulting Q(f) curves were found shifted and with same peak intensities. In a first approach and considering the outcomes of COLISEUM CEA model they were tentatively correlated by an homothetictransformation, i.e. an arbitrary factor applied on abscissa (frequencies) for MAG42-2 sample.

This gave very good results (see Fig. 7) by considering an homothety factor of 1.25 applied on MAG42-2 curve. 


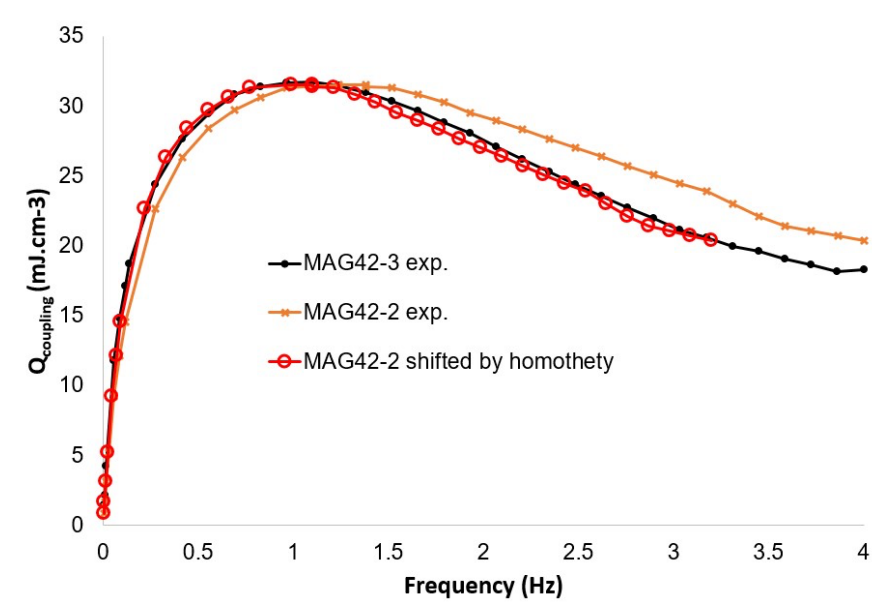

Fig. 7. MAG42-2 and MAG42-3 coupling losses Q(f) curves including MAG42-2 shift with a 1.25 homothety factor.

The 1.25 homothety factor relates in Fig. 7 to the frequency variable. However, in sinusoidal regime, the losses driver is the product $\{$ frequency $\mathrm{x}$ time constant $\}$ so the factor can freely be attributed to time constant. Since in classical models CICC time constant is proportional to inter-strand conductance, then it comes that the homothety factor in frequency can be attributed to conductance.

This means that for a relative matching of MAG42-2 and MAG42-3 coupling losses, a $25 \%$ conductance increase should be considered. This statement turns out to be perfectly in agreement with the outcomes of contact width statistic shown in section III.B.3) that claimed for a very close $26 \%$ conductance increase.

This very good fit consolidates the reliability of both databases (experimental tests and contact statistics) but also strengthen the robustness of interpretative underlying hypotheses (multi-constant approach in COLISEUM and interstrand contact widths relevancy to conductance) which is a satisfactory achievement. To further consolidate the present outcomes broader statistics should be also continued on other available samples, with other void fractions or also on other types of CICCs.

\section{B. Comparison on triplets}

The association of triplets proposed in section III.B.4) was checked versus extremities using the visual approach presented in section III.B.2), except that in the present case the strands nature is checked in every triplet $(2 \mathrm{NbTi}+1 \mathrm{Cu}$ association expected) and conservation of strands proximity in space is also controlled. On one hand it can be seen in first slice (Fig. 8) that all triplets obey the expected composition, which support our determination method. On the other hand in the last slice (Fig. 9), beyond the triplets composition, found correct, their spatial integrity (members stay close to each other) was also verified. This further strengthen our methodology, also keeping us confident that it can also be applied to the superior stages of the cable, up to its last stage.

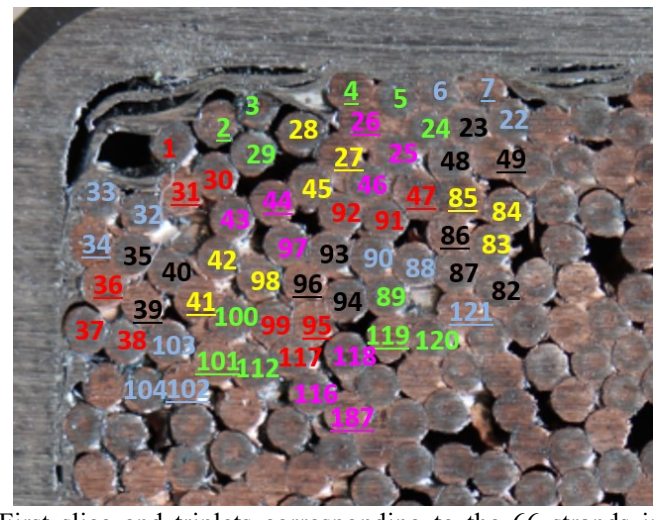

Fig. 8. First slice end triplets corresponding to the 66 strands in Fig. 5. Copper strands numbers are underlined. Color code variety is for triplets distinction purpose only.

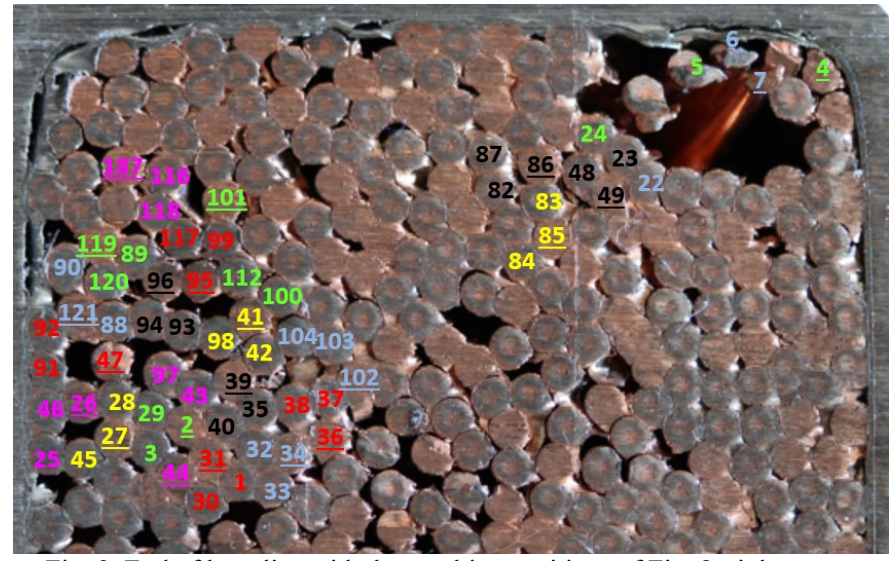

Fig. 9. End of last slice with the resulting positions of Fig. 8 triplets.

\section{Conclusion - Perspectives}

In a collaborative framework between CEA and INFLPR we developed a global analysis workflow dedicated to the reconstruction of a CICC $3 \mathrm{D}$ geometry associated with an analysis protocol oriented towards inter-strand contact statistics. The associated tools and methods were established and their content consolidated by dedicated checks on two samples with varying void fraction.

The analysis conducted on the two samples showed a remarkable consistency between the outcomes of contact statistics and coupling losses tests results. Besides, a promising method for cable structure reconstruction was initiated, aiming at providing in the future relevant inputs for coupling models such as COLISEUM.

As per future investigations broadening issued from this work, many paths are still to be explored, among which: enlarging statistics and coupling losses checks with other cables having different features (void, pattern, size); building the 3D inter-strand electrical network for $\mathrm{AC}$ and $\mathrm{DC}$ regimes modelling purpose ; using CICC strands 3D data on intricated geometry for mechanical modelling; using CICC 3D void topology for modelling hydraulic properties prediction (fluid friction \& heat exchange). Those items will be subject of future communications. 


\section{REFERENCES}

[1] M.O. Hoenig, "Internally cooled cabled superconductors", Cryogenics Vol. 20, n7, pp. 373-389 (1980)

[2] Y. Koide et al., "JT-60SA Superconducting Magnet System", Nucl. Fusion Vol. 55, p. 086001 (2015)

[3] A Devred et al., Supercond. Sci. Technol. Vo. 27 Art. 044001 (2014).

[4] W. Chen, "Fabrication of the Toroidal Field Superconducting Coils for the EAST Device", IEEE Trans. Appl. Supercond., Vol. 16, n² (2006).

[5] L. Zani, P. Barabaschi and M. Peyrot, "Starting EU production of strand and conductor for JT 60SA Toroidal Field coils", IEEE Trans. Appl. Supercond. Vol. 22 n³, p.4801804 (2012)

[6] I. Tiseanu et al., "Characterization of Superconducting Wires and Cables by X-ray Micro-Tomography", Fus. Engin. and Design. Vol. 88, n ${ }^{\circ} 9-10$ pp 1613-1618 (2013)

[7] I. Tiseanu, L. Zani, C. Tiseanu, T. Craciunescu, C. Dobrea, "Accurate 3D modeling of Cable in Conduit Conductor type superconductors by X-ray microtomography", Fusion Engineering and Design, Vol. 98-99, Pages 1176-1180 (2015)

[8] L. Zani et al., "Influence of Strands Trajectories of JT-60SA TF Conductors on their Hydraulic and Electromagnetic Properties", IEEE Trans. Appl. Supercond. Vol.26, n 4, p. 4802405 (2016)

[9] Tiseanu I. et al., "Multi-scale 3D modelling of a DEMO prototype cable from strand to full-size conductor based on X-ray tomography and image analysis", Fus. Engin. and Sesign, Vol. 146, pp. 568-573 (2019)

[10] L. Zani et al., "Progresses at CEA on EU DEMO reactor cryomagnetic system design activities and associated R\&D”, Nuclear Fusion, Vol. 59 Art. 086033 (2019)

[11] F. Aurenhammer, R. Klein and D-T. Lee, "Voronoi Diagrams And Delaunay Triangulations", World Scientific Publishing Company (2013). ISBN 978-981-4447-65-2.

[12] M. Chiletti et al., "Analytical Modelling of CICCs Coupling Losses: Broad Investigation of Two-Stage Model", IEEE Transactions on Applied Superconductivity Vol.29, n5, Art. 4703005 (2019)

[13] M. Chiletti et al., Analytical coupling losses modelling with COLISEUM: generalized approach upgraded to n stages, presented at ASC 2020, to be submitted to IEEE Trans. Appl. Supercond.

[14] M. Chiletti et al. "Void fraction influence on CICCs coupling losses: analysis of experimental results with MPAS model", IEEE Trans. Appl. Supercond. Vol. 30 n $^{\circ} 4$, Art. $\mathrm{n}^{\circ} 4701405$ (2020) 